\title{
Um Olhar sobre o Processo de Design e Avaliação do VINI sob a Perspectiva do Processo de Design Inclusivo
}

\author{
Rallyson dos S. Ferreira \\ Universidade Federal do \\ Amazonas(UFAM) \\ Itacoatiara-AM, Brasil \\ rallysonferreira@hotmail.com
}

\author{
Thaís H. Chaves de Castro \\ Universidade Federal do \\ Amazonas(UFAM) \\ Manaus-AM, Brasil \\ thais@icomp.edu.br
}

\author{
David Lima \\ Universidade Federal do \\ Amazonas(UFAM) \\ Manaus-AM, Brasil \\ david.lima@icomp.edu.br
}

\author{
Marcelino Pereira de Sena Jr \\ Universidade Federal do \\ Amazonas(UFAM) \\ Itacoatiara-AM, Brasil \\ marcelinosena@outlook.com
}

\begin{abstract}
RESUMO
Nas últimas décadas a sociedade brasileira tem enfrentado um grande desafio, que é a interação em ambientes pensados e planejados para crianças neurotípicas, principalmente, o Transtorno do Espectro Autista (TEA) [3], [8]. Sabendo que o uso da conhecida metodologia TEACCH (Treatment and Education of Autistic and Communication Handicapped Children) [4] vem se mostrando eficaz para tranquilizar e orientar crianças com o TEA, foi implementada uma aplicação móvel denominada VINI para automatizar o processo de criação de atividades segundo essa metodologia. Sendo assim, foram aplicados métodos e técnicas para design e avaliação da interação de crianças com esse aplicativo. Ao longo do processo avaliativo [5], ao analisar a experiência de uso do VINI, foram percebidas necessidades de se utilizar métodos que se adaptassem aos novos contextos e perfis desses usuários, pois ainda que sejam crianças com autismo em fase escolar, suas necessidades específicas sobressaem qualquer perfil diagnóstico. Dessa forma, foi realizada uma releitura dos processos de design e avaliação sob o olhar do Processo de Design Inclusivo (PDI) [6] visando identificar falhas no processo quanto a possíveis individualizações de contexto desde as primeiras fases do design.
\end{abstract}

\section{Palavras-chave}

Transtorno do Espectro Austista; TEACCH, Processo de Design Inclusivo; Design de Interação.

Permission to make digital or hard copies of all or part of this work for personal or classroom use is granted without fee provided that copies are not made or distributed for profit or commercial advantage and that copies bear this notice and the full citation on the first page. Copyrights for components of this work owned by others than the author(s) must be honored. Abstracting with credit is permitted. To copy otherwise, or republish, to post on servers or to redistribute to lists, requires prior specific permission and/or a fee. Copyright 2018 SBC.

IHC 2018, Anais Estendidos do XVII Simpósio Brasileiro sobre Fatores

Humanos em Sistemas Computacionais

Outubro 22-26, 2018, Belém, Brasil

PÔSTERES VIRTUAIS E DEMOS
ACM Classification Keywords

J.1.1 [Human-centered computing]: Human computer interaction (HCI) - HCI design and evaluation methods, Interaction design (ID) - Interaction design process and methods.

\section{DEFINIÇÃO DO TRABALHO}

O PDI é utilizado neste projeto no desenvolvimento de uma aplicação denominada VINI, o qual foi projetado seguindo os preceitos da metodologia multidisciplinar de alfabetização TEACCH, que tem apresentado resultados bastante animadores, favorecendo uma compreensão de mundo a crianças com TEA [4].

Durante o processo de avaliação da ferramenta [5], a usabilidade apontou falhas no uso que poderiam ser evitadas se fossem consideradas as habilidades específicas de crianças com autismo. Com isso, surgiu a necessidade de reavaliar todo o processo de design e avaliação do VINI utilizando um olhar de design diferenciado do PDI [6].

As etapas do PDI foram observadas e simuladas nessa releitura e pressupostos levantados para reduzir os erros de design no sentido de reprojetar o app de forma que seja possível adaptá-lo, desde as primeiras fases do design, às necessidades ou habilidades individuais de cada criança e professor.

\section{PROCESSO DE REENGENHARIA}

O aplicativo VINI se apoia na utilização de técnicas de aprendizagem sem valorização do erro, que auxilia a criança a perceber o que é possível ou não dentro de suas ações no ato de execução da atividade.

Sendo assim, nessa mesma perspectiva o app apresenta outra característica importante, todos os campos estão configurados de forma que acionem dicas de acordo com o número de erros que a criança comete ao realizar uma atividade. Além das dicas, o jogo também possui diversos objetos e palavras, apresentando tutoriais por todos os níveis. 
O processo de reconstrução do design do VINI foi baseado, principalmente, no feedback de todos os envolvidos no processo de design, pais, crianças com TEA e profissionais. Esse feedback foi em forma de questionários respondidos pelos pais, seguindo recomendações de [2] e do processo de design inclusivo [3], [6], [7], o que gerou uma releitura do processo de design para novos artefatos.

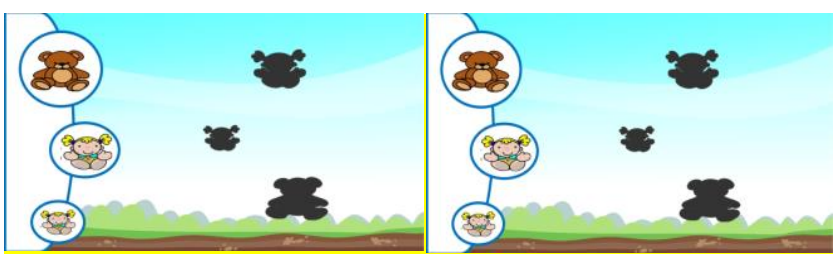

Figura 1. Forma e tamanho dos objetos [2].

A Figura 1 mostra uma das modificações desenvolvidas, seguindo o feedback dos pais e profissionais que é a variabilidade da forma e tamanho dos objetos (uma modificação por vez).

Quanto à releitura seguindo o processo de design inclusivo, foi identificada a necessidade de testar pressupostos da psicopedagogia que as crianças estavam sendo expostas já nos primeiros protótipos elaborados com a equipe multidisciplinar para que fossem inseridos os elementos adaptativos gradativamente e ao mesmo tempo capturassem uma maior quantidade de perfis cognitivos. Aplicado ao design do VINI, o PDI auxilia a evitar falhas de inadequabilidade e ajuste fino no app que só foram identificados na fase de avaliação, através dos questionários respondidos pelos pais e profissionais e exploração livre pelas crianças com TEA.

Ainda que essa releitura tenha sido realizada sobre o VINI, outros artefatos computacionais pensados e projetados para crianças com autismo e outras deficiências intelectuais podem se beneficiar. Ainda há a possibilidade de ampliar o escopo de apps assistivos introduzindo os pressupostos de design e usabilidade universais, o que já está sendo realizado no contexto desse app, aplicado a outros artefatos assistivos.

\section{CONSIDERAÇÕES}

O desenvolvimento do VINI, seguindo os preceitos do processo de design inclusivo, fortalece uma linha de ação dentro de acessibilidade e IHC, em relação a como atender melhor os propósitos terapêuticos e/ou pedagógicos essa necessidade de se projetar com e para crianças e adolescentes com deficiência intelectual. Profissionais que projetam atividades diariamente para essas crianças e adolescentes assim como os familiares possuem uma demanda forte por artefatos computacionais que reforcem as terapias e conteúdo escolar.

Dessa forma, esses artefatos podem ser úteis também para auxiliar na inclusão de crianças e adolescentes com TEA no ensino regular, sendo pensados já de início como um PDI, utilizando design e usabilidade universais para que todos de uma mesma turma utilizem.
Outra consequência é o desenvolvimento de tecnologias adaptáveis, ou seja, tecnologias que os usuários possam modificar para atender às necessidades de cada indivíduo ou mesmo de um grupo de indivíduos [1]. Assim, ao possibilitar que uma tecnologia possibilite ao profissional, que crie atividades personalizadas para todos os seus pacientes ou alunos isso contribuirá para a inclusão dessas pessoas [8].

\section{REFERÊNCIAS}

1. Pricila Braz, Clarisse S. de Souza, Alberto Raposo. 2014. Design de Tecnologias Adaptáveis para Uso de Profissionais da Área de Autismo. Brazilian Symposium on Human Factors in Computing Systems. October 27-31, 2014, Foz do Iguaçu, PR, Brazil.

2. Talita C. P. Britto, Ednaldo B. Pizzolato. 2016. Gaia: uma proposta de um guia de recomendações de acessibilidade de interfaces web com foco em aspectos do autismo. V Cngresso Brasileiro de Informática na Educação (CBIE 2016).

3. Thaís Castro, David Lima. 2017. Designing for Children Using the RtD and HCD Approaches. In: Antona M., Stephanidis C. (eds) Universal Access in Human-Computer Interaction. Design and Development Approaches and Methods. UAHCI 2017. Lecture Notes in Computer Science, vol 10277. Springer, Cham.

4. Maria E. Fonseca, Juliana C. Ciola. 2014. Vejo e Aprendo: Fundamentos do Programa TEACCH. O Ensino Estruturado para Pessoas com Autismo. $1^{\circ}$ edição. Book Toy.

5. Jacob Nielsen, Rolf Molich. 1994. Heuristic Evaluation. New York.

6. Ulrike Lucke, Thais Castro. 2016. The Process of Inclusive Design. In IEEE 16th International Conference on Advanced Learning Technologies. pp. 446-447.

7. Filomena M. G. S. C. Moita, Lucas H. Viana, Felipe M. Medeiros, Vilma M. A. Candido. 2017. Design e desenvolvimento de um game assistivo para autistas. VI Cngresso Brasileiro de Informática na Educação (CBIE 2017).

8. Fernando C. B. G. Santana, Lucas R. de Carvalho, Gueilherme N. S. Ribeiro, Sandra E. V. Aguiar, Ana C. R. Santana. 2017. SATA (Sistema de Acompanhamento do Tratamento de Autismo). VI Cngresso Brasileiro de Informática na Educação (CBIE 2017). 